\title{
Application of Responsive Neurostimulation as both a Diagnostic Tool for Seizure Localization and a Supplementary Tool for Surgical Management in Patients with Multiple Epileptogenic Foci: A Case Series
}

\author{
Kamran Urgun, Alvin Chan, Ronald Sahyouni, Kieu Tran, Frank Hsu, Sumeet Vadera \\ University of California, Irvine Medical Center, Department of Neurological Surgery, Orange, CA, USA
}

Corresponding author: Kamran URGUN drkamranurgun@gmail.com, kurgun@hs.uci.edu

\section{ABSTRACT}

AIM: To present a series of medically refractory focal epilepsy patients with multiple or eloquent epileptogenic zones (EZs) in whom a responsive neurostimulation (RNS) system was used as a complementary modality to surgical resection. RNS was also used as a diagnostic tool to monitor long-term epileptogenic activity for enhanced localization, especially in patients with bilateral temporal seizures.

MATERIAL and METHODS: Ten consecutive patients who underwent RNS system placement and surgical resection at a single institution were assessed.

RESULTS: The RNS system, with its capacity for chronic ambulatory electrocorticography (ECoG), provided important diagnostic information that helped to modify the plan of surgical resection in one patient with bitemporal epilepsy in order to improve seizure outcomes. In addition, the RNS facilitated the surgical management of patients with multiple or eloquent EZs.

CONCLUSION: The authors report a population of 10 patients in which the RNS system was used as a diagnostic tool for improved localization of EZs over a long interval or as a complementary therapeutic tool in patients with multiple or eloquent EZs.

KEYWORDS: Responsive neurostimulation, Robot enhanced neurosurgery, Epileptogenic zone

\section{INTRODUCTION}

$\mathrm{P}$ atients receiving medical management of medically refractory focal epilepsy often benefit from surgical resection of the epileptogenic zone (EZ) if precisely localized (14). However, in patients with medically refractory epilepsy who are not good candidates for surgical resection due to multiple or eloquent EZs, neuromodulation methods such as vagus nerve stimulation, responsive neurostimulation (RNS) (NeuroPace Inc., Mountain View, CA) and deep brain stimulation have been applied safely as therapeutic options $(1,5)$.
RNS has been used as an adjunctive treatment in patients with partial-onset epilepsy arising from eloquent cortical areas or patients with multiple ictal onset zones. The goal is to provide a reduction in seizure burden when surgical resection is unsafe or unviable (e.g., bilateral mesial temporal lobe epilepsy or an onset zone in an eloquent area) $(2,12)$. In addition to continuous monitoring activity and delivering predetermined electrical pulses to interrupt developing epileptogenic activity, RNS provides diagnostic information for seizure lateralization that may help clinicians tailor the management in favor of surgical intervention at a later date $(3,6,8,11,13)$.
Kamran URGUN (10): 0000-0002-1857-8797

Alvin CHAN (D): 0000-0002-0952-4367

Ronald SAHYOUNI (1) : 0000-0002-2124-0535 $\begin{array}{ll}\text { Kieu TRAN } & \text { (1) : }: 0000-0002-6788-0239 \\ \text { Frank HSU } & \text { (1) : 0000-0003-0929-4052 } \\ \text { Sumeet VADERA (i) : }: 0000-0002-1501-542 X\end{array}$ 
In this study, we describe the use of RNS to characterize and localize seizure activity in chronic ambulatory monitoring as well as a complementary tool for patients undergoing surgical resection. To our knowledge, this is the first case series of outcomes related to patients undergoing resection and RNS.

\section{MATERIAL and METHODS}

With institutional review board approval, a retrospective electronic chart (EPIC Systems, Verona, WI) review was performed evaluating patients between June 2016 and April 2019 who underwent both RNS implantation and surgical resection after invasive intracranial (either stereoelectroencephalography (SEEG) or SDG placement) monitoring. Patients with epilepsy whose EZs are wide or eloquent enough for resection or in whom the RNS may not be able to cover all EZ zones were offered RNS placement and surgical resection in the same session after phase II monitoring. The surgical procedure, perioperative risks, and challenges in the long-term follow-up were discussed in detail.

Only one of those patients had surgical resection several months later after evaluating chronic ambulatory recordings obtained from the RNS, and in the remaining 9 patients, the RNS systems were implanted in the same session with resective surgical intervention including temporal lobectomy.

All phase II interventions ('SEEG'in 5 patients and 'SEEG + SDG' in 3 patients) and implantations of RNS electrodes were carried out with framed stereotactic guidance with robot assistance (ROSA, MedTech, Montpellier, France) (4). Noninvasive monitoring (video electroencephalography) showed bilateral independent ictal onset in one patient; therefore, a phase II intervention was not necessary.

\section{RESULTS}

Fourteen patients were found who underwent RNS placement plus resection of EZ, but 4 of them were excluded since their follow-up was less than 6 months. A total of 10 patients (5 females and 5 males) with an average age of 35.1 years (range: 27-53 years, median: 31 years) and an average onset of seizure 12 years (range: 4-49 years, median: 12 years) with epilepsy at the time of therapeutic intervention were identified, all of whom had undergone both RNS implantation and surgical resection in the management of epileptogenic foci with an average follow-up of 32 months (range: 6-44 months, median: 17 months).

All patients underwent extensive monitoring for EZ through noninvasive and invasive methods. In all patients, surgical resection was performed in the same session with RNS implantation or several months later after the analysis of chronic monitorization from the RNS showed better characterization and localization of the epileptogenic sources. Tables I and II show the summary of demographic properties, the pre- and postoperative workflow and postop complications for those

Table I: Summary of 10 Patients with Intractable Epilepsy Who Underwent RNS System Implantation and Surgical Resection

\begin{tabular}{|c|c|c|c|c|c|c|c|c|}
\hline $\begin{array}{l}\text { Patient } \\
\text { No. }\end{array}$ & $\begin{array}{l}\text { Age (y), } \\
\text { Sex }\end{array}$ & $\begin{array}{c}\text { Average } \\
\text { Duration (y) }\end{array}$ & $\begin{array}{l}\text { History of } \\
\text { Previous } \\
\text { Surgeries }\end{array}$ & $\begin{array}{c}\text { Preoperative } \\
\text { Number of } \\
\text { AEDs }\end{array}$ & $\begin{array}{l}\text { Number of } \\
\text { AEDs at the } \\
\text { Last Follow-Up }\end{array}$ & $\begin{array}{l}\text { Preoperative } \\
\text { Seizure } \\
\text { Frequency }\end{array}$ & $\begin{array}{c}\text { Seizure } \\
\text { Frequency } \\
\text { at the Last } \\
\text { Follow-Up }\end{array}$ & $\begin{array}{c}\text { Result } \\
\text { (Seizure } \\
\text { Freedom) }\end{array}$ \\
\hline 1 & $31, F$ & 12 & - & 5 & 4 & 5/month & $1 /$ month & IIIA \\
\hline 2 & $33, F$ & 15 & $\begin{array}{l}\text { Left-sided } \\
\text { craniotomy due to } \\
\text { traumatic injury }\end{array}$ & 4 & 4 & Daily & - & IA \\
\hline 3 & $28, M$ & 27 & - & 4 & 3 & Daily & 2/week & IIIA \\
\hline 4 & $27, \mathrm{~F}$ & 4 & - & 4 & 4 & Daily & $2 /$ month & IIB \\
\hline 5 & $33, \mathrm{M}$ & 11 & $\begin{array}{l}\text { Encephalomalacia } \\
\text { in the bilateral } \\
\text { frontal areas } \\
\text { due to traumatic } \\
\text { encephalopathy }\end{array}$ & 3 & 3 & 6/month & - & IA \\
\hline 6 & $53, \mathrm{M}$ & 12 & - & 3 & 1 & 3/week & - & IA \\
\hline 7 & $41, M$ & 8 & - & 2 & 3 & $2 /$ month & $\begin{array}{c}\text { Only } 2 \\
\text { seizures }\end{array}$ & IIA \\
\hline 8 & $49, \mathrm{~F}$ & 49 & VNS implantation & 3 & 3 & 2/week & - & IA \\
\hline 9 & $25, \mathrm{M}$ & 6 & - & 3 & 3 & Several/month & $\begin{array}{l}\text { Only } 4 \\
\text { episodes }\end{array}$ & IIB \\
\hline 10 & $31, F$ & 26 & $\begin{array}{l}\text { Right frontal area } \\
\text { resection + VNS } \\
\text { implantation }\end{array}$ & 3 & 3 & $6 /$ month & - & IA \\
\hline
\end{tabular}




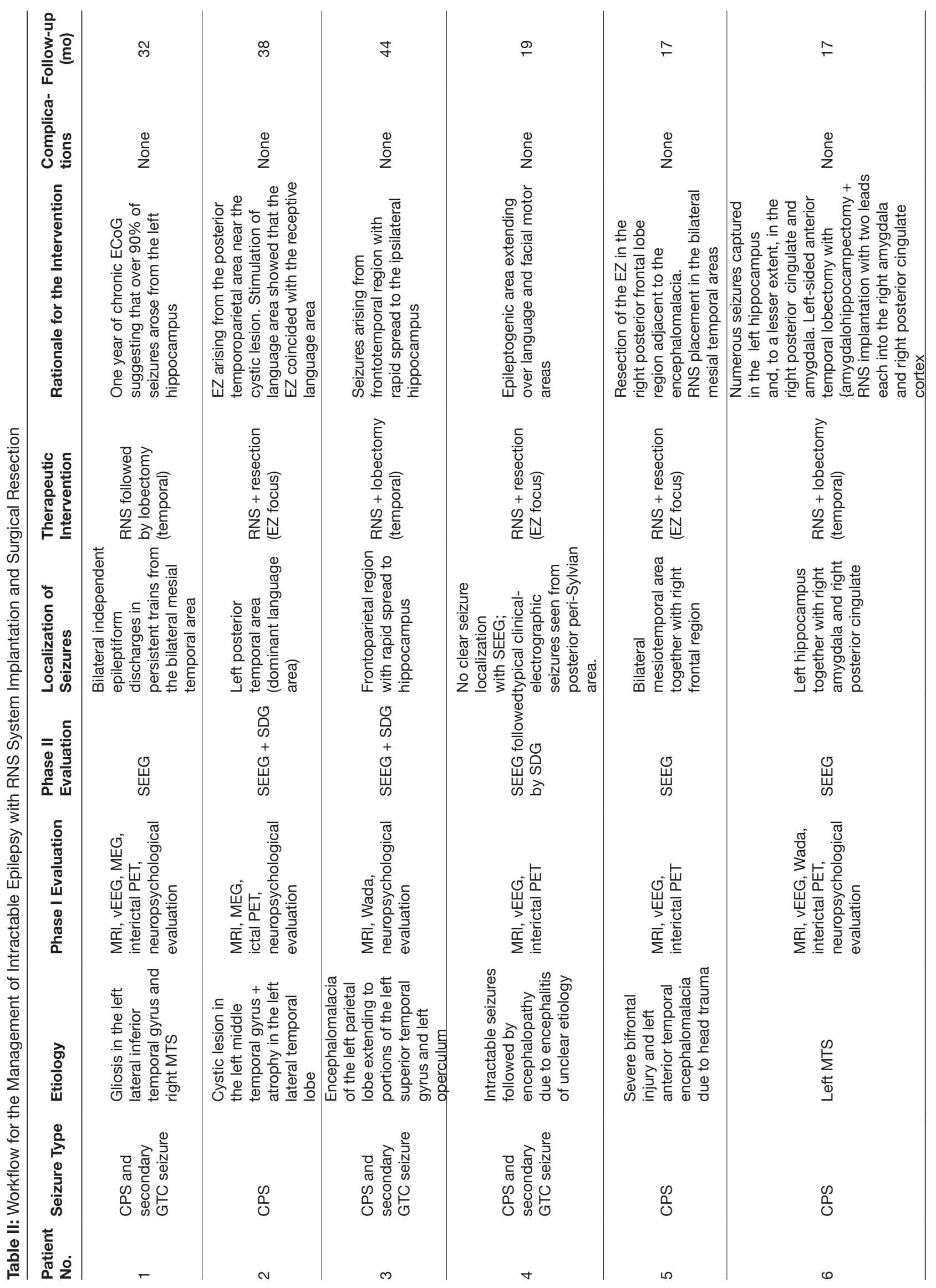




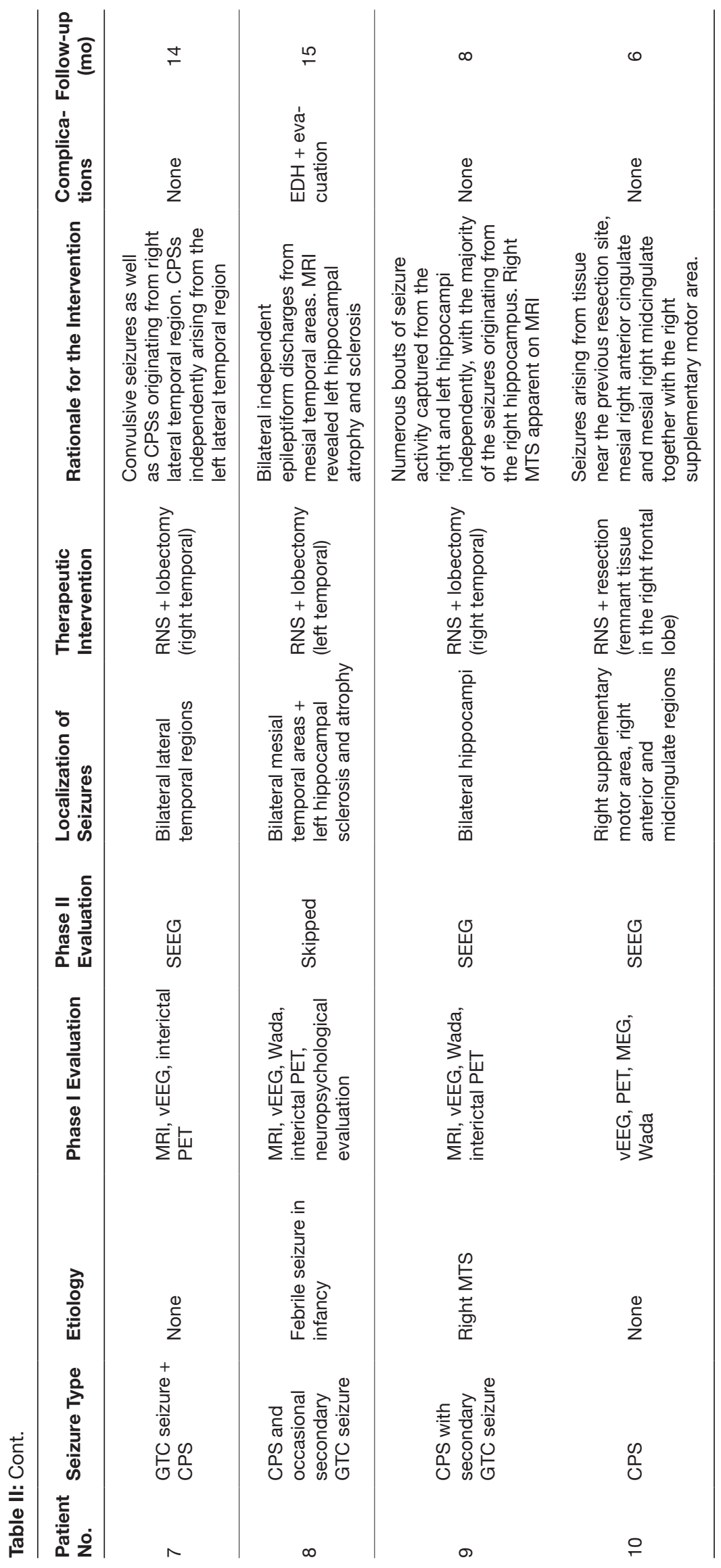


patients detailed below. In this paper, the Engel Epilepsy Surgery Outcome Scale was used to classify outcome after surgical treatment for medically refractory outcome (7).

The patient in Case 1 was a right-handed 31-year-old female with a history of suspected encephalitis and refractory epilepsy for the last 12 years who was not considered a suitable candidate for resective surgery since SEEG showed bilateral independent mesial temporal seizure onset. An RNS system was implanted with bilateral depth electrodes, and one year of chronic ECoG recording from the RNS demonstrated that over $80 \%$ of her seizures arose from the left hippocampus without meaningful improvement with RNS treatment with respect to frequency. Subsequently, a left anterior lobectomy with amygdalohippocampectomy was performed, and the patient has been followed for 32 months since the lobectomy with over $50 \%$ reduction of her initial seizures.

The patient in Case 2, a right-handed 33-year-old female with a history of a left-sided craniotomy due to traumatic brain injury, subsequently developed medically refractory complex partial seizures for the last 15 years. She had also undergone extensive workup at another facility showing seizures all lateralizing to the left without any exact localization (Figure 1A). The patient was discussed at a multidisciplinary epilepsy conference and underwent left-sided subdural grid and depth electrode placement (Figure 1B). Chronic monitoring demonstrating seizures arising from the left posterior temporoparietal areas. After stimulating the language area, the patient was found to have her EZ overlap with her receptive language area. She eventually underwent a limited, partial resection excluding the receptive language area and placement of the RNS system with paddle electrodes in the remaining EZ areas (Figure 2A,B). She has been followed for 38 months since her therapeutic surgery, and she is seizure free.
The patient in Case 3, a right-handed 28-year-old male with a history of complex partial seizures since the age of 1 year, underwent chronic invasive monitoring with both subdural grids and depth electrodes. Seizures were determined to originate from the left frontotemporal area with rapid spread to the left hippocampus. Therefore, the patient underwent a leftsided temporal lobectomy with amygdalohippocampectomy and implantation of the RNS system with placement of paddle electrodes over the left frontal area. The patient has been followed for 44 months since his therapeutic intervention, and his seizure frequency decreased from multiple seizures per day down to two per week together with a decrease in the number of medications and dosages.

The patient in Case 4, a right-handed 27-year-old female, had a history of drug-resistant localization-related epilepsy for the last 4 years developing after a viral illness and had undergone invasive monitoring with bilateral depth electrodes covering bilateral mesiotemporal regions, insulae, anterior and posterior cingulate regions, peri-Rolandic and supplementary motor area (SMA) without clear localization but lateralization to the left hemisphere. She underwent left-sided subdural grid placement over the peri-Rolandic area, SMA and lateral temporal convexity that captured clinical-electrographic seizures with the best ictal pattern seen from the peri-Sylvian area (Figure 3A). Cortical mapping was performed to define surgical margins, with language area and face/arm areas identified on the grid map (Figure 3B). After discussion with the patient, she underwent RNS placement with two paddle electrodes covering the language area and neocortical resection anterior to the contacts involved in the language area (Figure 4A-D) in the same session. She was suffering daily seizures that dropped to two per month at her 19-month follow-up.
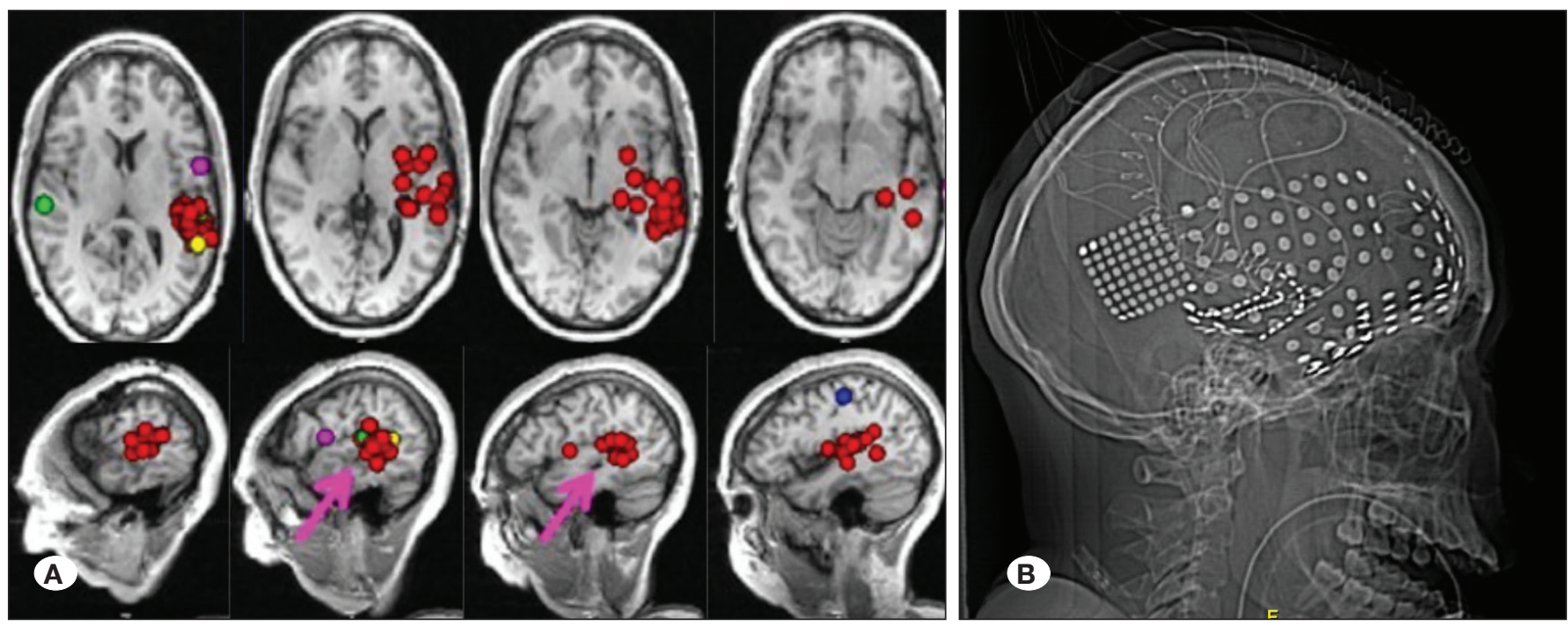

Figure 1: A) An MEG study showed clusters of spikes in the posterior temporoparietal region near Wernicke's area as well as spikes in the insula. (Pink: expressive language area; yellow: receptive language area; blue: median nerve response; red: spikes; green: auditory response. Pink arrows show the area of clustering of the spikes over Wernicke's area.) B) Lateral radiographic image with localization of left-sided subdural grids and depth electrodes. 

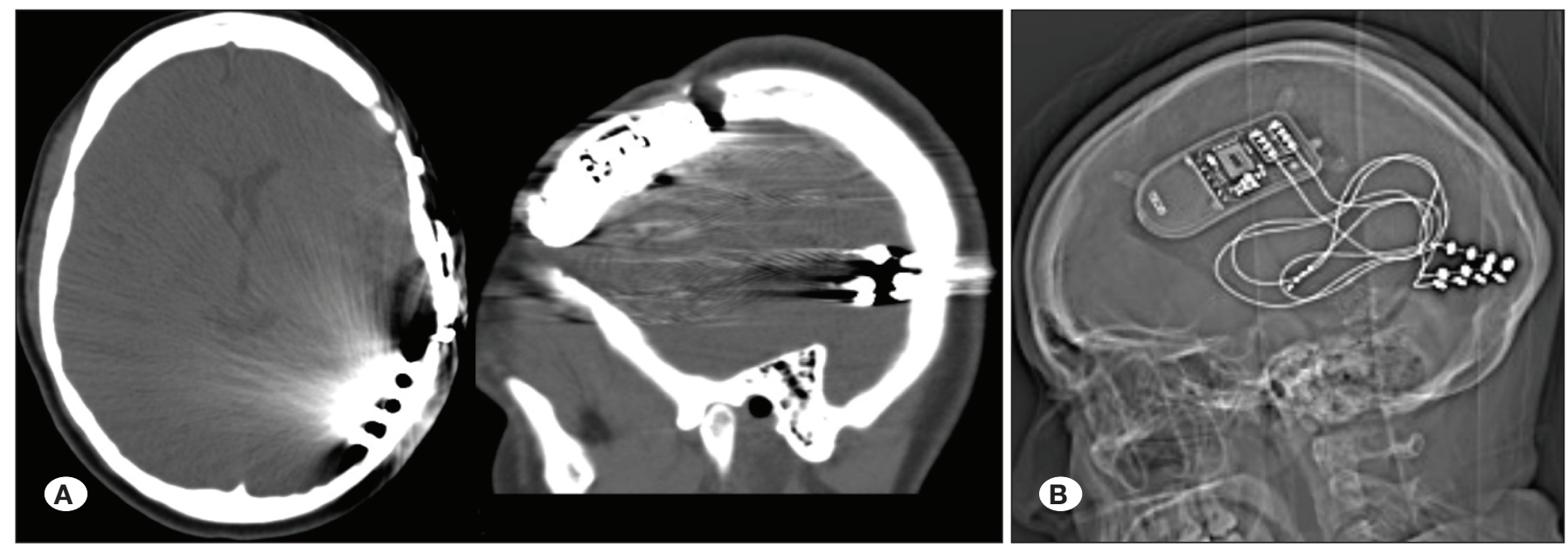

Figure 2: Cranial CT (A) shows the resected area, and the lateral radiographic image (B) shows paddle electrodes over the receptive language area.
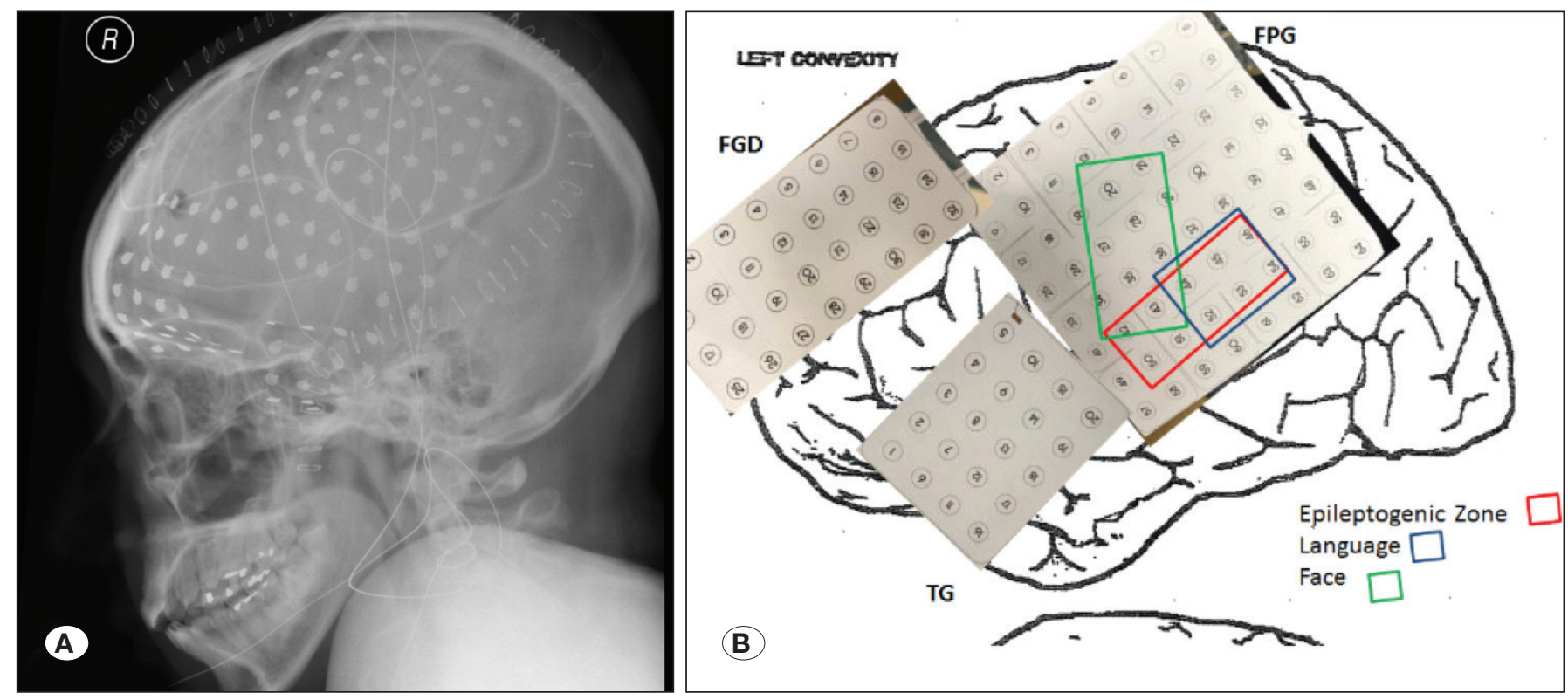

Figure 3: Lateral radiographic image (A) showing the localization of left-sided subdural grids and cortical mapping, and (B) indicating the language area and face/arm area in relation to the EZ.

The patient in Case 5 was a right-handed 33-year-old male with a history of severe traumatic brain injury who developed medically intractable epilepsy four years after the injury. Chronic monitoring with multiple depth electrode SEEG (Figure $5 A$ ) revealed that the seizures were arising predominantly from the right frontal lobe adjacent to the previously injured area and intermittently from bilateral temporal regions, lateral on the left and mesial on the right. After discussion, it was decided that the patient was a candidate for a right posterior frontal lobe resection of the EZ adjacent to the injured area and placement of RNS placement in the bilateral temporal region (Figure $5 B$ ). He was seizure free at the end of a 17-month follow-up period after his therapeutic surgery.
The patient in Case 6, a right-handed 53-year-old male, had a history of medically refractory localization-related epilepsy whose cranial MRI demonstrated left-sided mesial temporal sclerosis (MTS), and PET scan showed nonspecifically increased metabolism in the left parahippocampal gyrus and right parietal region. During chronic monitoring, numerous seizures were captured in the left hippocampus and to a lesser extent in the right hemisphere, more precisely from the depth electrodes in the right posterior cingulate and right amygdala. Given the out-of-proportion seizures from the left hippocampus plus RNS implantation with two leads, he underwent a left-sided anterior temporal lobectomy with amygdalohippocampectomy. At his 17-month follow-up, he was seizure free with a reduced number of antiepileptic medications. 

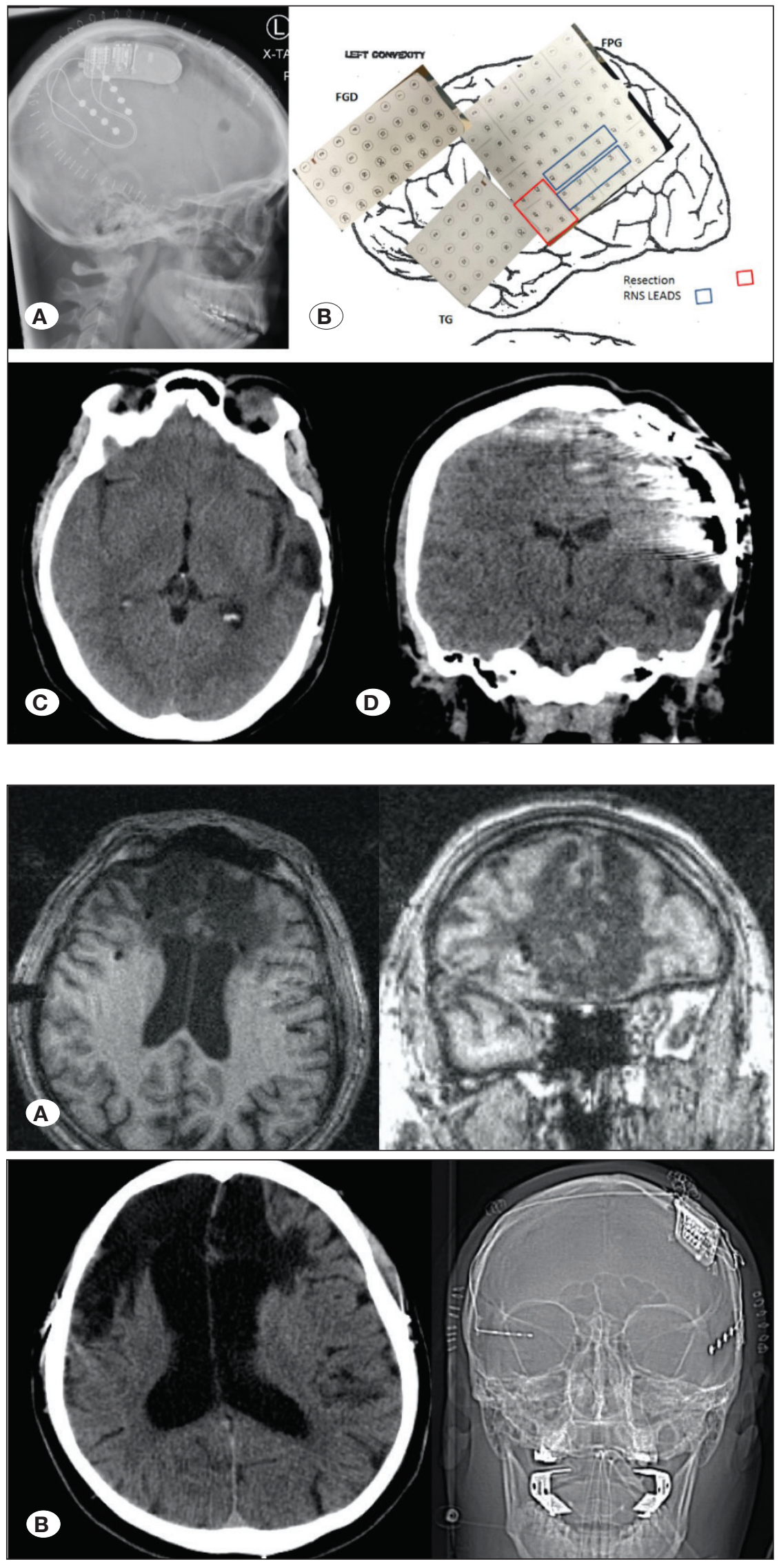

Figure 4: The small neocortical area to be resected based on cortical mapping (A). Lateral radiographic image $(\mathbf{B})$ that shows the location of RNS implantation, with its paddle electrodes over the EZ. Axial and coronal cranial CT images (C, D) show the resected area and artifacts of the paddle electrodes over the remaining $E Z$.

Figure 5: Cranial MRI obtained in the early post-SEEG period showing bilateral frontal encephalomalacia resulting from traumatic brain injury. Several depth electrodes are also visible in the images (A). An axial cranial CT image and an anteroposterior (AP) radiographic image show the RNS system, with its bilateral temporal electrodes and area of resection in the right lateral frontal lobe adjacent to the area with encephalomalacia (B). 
The patient in Case 7 is a right-handed 41-year-old male with a history of medically intractable complex partial seizures with secondary generalization for the last 8 years. Chronic monitoring with intracranial depth electrodes demonstrated complex partial seizures originating from the left lateral temporal region with propagation to the left mesial temporal/ hippocampus area; subclinical seizures arising from the right lateral temporal area; and generalized tonic-clonic seizures originating from the right lateral temporal region, propagating to the right anterior temporal region and finally spreading to the right mesial temporal area. Therefore, a right-sided anterior temporal lobectomy together with RNS implantation into the left temporal area was performed (Figure 6). The patient was seizure free for several months and had only two episodes at the end of his 14-month follow-up.

The patient in Case 8, a left-handed 49-year-old female with a history of febrile seizure, started first in her infancy, and subsequent medically refractory complex partial seizures with secondary generalized tonic-clonic seizures had undergone vagus nerve stimulation (VNS) system placement at the age of 42 years. Cranial MRI demonstrated left-sided hippocampal atrophy and sclerosis. Noninvasive chronic monitorization showed that the patient had bilateral independent seizure onset from the bilateral temporal regions. After discussion, it was decided that she would benefit most from a left anterior temporal lobectomy with amygdalohippocampectomy and RNS system implantation with two depth electrodes (orthogonal and oblique) in the right mesial temporal region. On the day of the procedure, this patient developed an epidural hematoma under the craniectomy that was made for RNS battery placement; the hematoma was evacuated without further complications. The patient was discharged home on postoperative day 3 with no neurological deficits. She was seizure free throughout her 15-month follow-up period.

The patient in Case 9 is a right-handed 25-year-old right male with a history of two febrile convulsions in infancy and subsequent medically refractory complex partial epilepsy with secondary tonic-clonic progression since the age of 19 years. The patient needed frequent hospitalizations for most of the seizure episodes for a longer duration. A cranial MRI demonstrated right-sided mesial temporal sclerosis, and long-term noninvasive vEEG monitoring was consistent with bilateral, multifocal epilepsy with more than 60 stereotyped seizures captured. Although the majority of the patient's seizures originated from the right temporal area, since some of the seizures were suggestive of contralateral onset, including one seizure that progressed to prolonged bilateral tonic-clonic activity, the patient underwent invasive continuous monitorization with depth electrodes eventually to further localize epileptogenic focus or foci. SEEG was diagnostic of focal epilepsy of independent left and right hippocampal origin, with the majority of seizures originating from the right hippocampus. Therefore, he was considered a good candidate for a right anterior temporal lobectomy with amygdalohippocampectomy together with RNS implantation in the left hippocampus in the same session. He had had only four seizure episodes by his 8-month follow-up, all of them shorter in duration than his previous episodes. A review of his last electrocorticography (ECoG) data at the 8-month follow up revealed a total of 170 long seizure episodes (i.e., lasting more than 30 seconds) in the last 3 months, with an average of 52.4 seizures detected per month.

The patient in Case 10, a right-handed 31-year-old female with medically refractory frontal lobe epilepsy beginning at the age of 5 years, had undergone right lateral frontal resection and VNS system placement in another facility (Figure 7A). Chronic invasive monitoring with bilateral depth electrodes demonstrated electroclinical seizures arising from the right mesial frontal region, including anterior and middle cingulate areas, with a variable amount of delay time prior to seeing the low-voltage fast activity, and tonic-clonic seizures with fast activity arising from the right supplementary motor and right

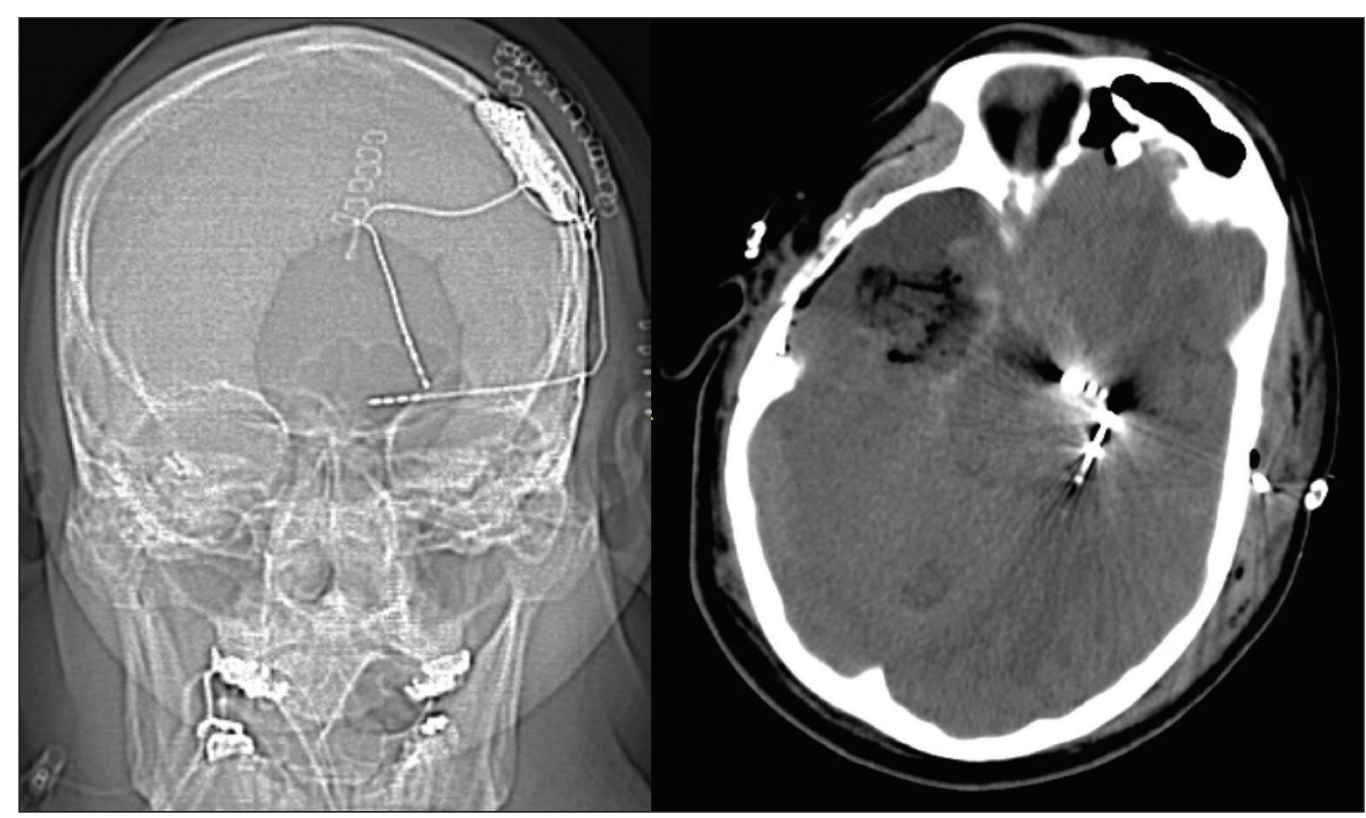

Figure 6: Cranial CT shows the resection cavity from the right-sided temporal lobectomy (right), and the anteroposterior (AP) radiographic image shows the two depth electrodes (orthogonal and oblique) of the RNS system implanted in the right mesial temporal region (left). 

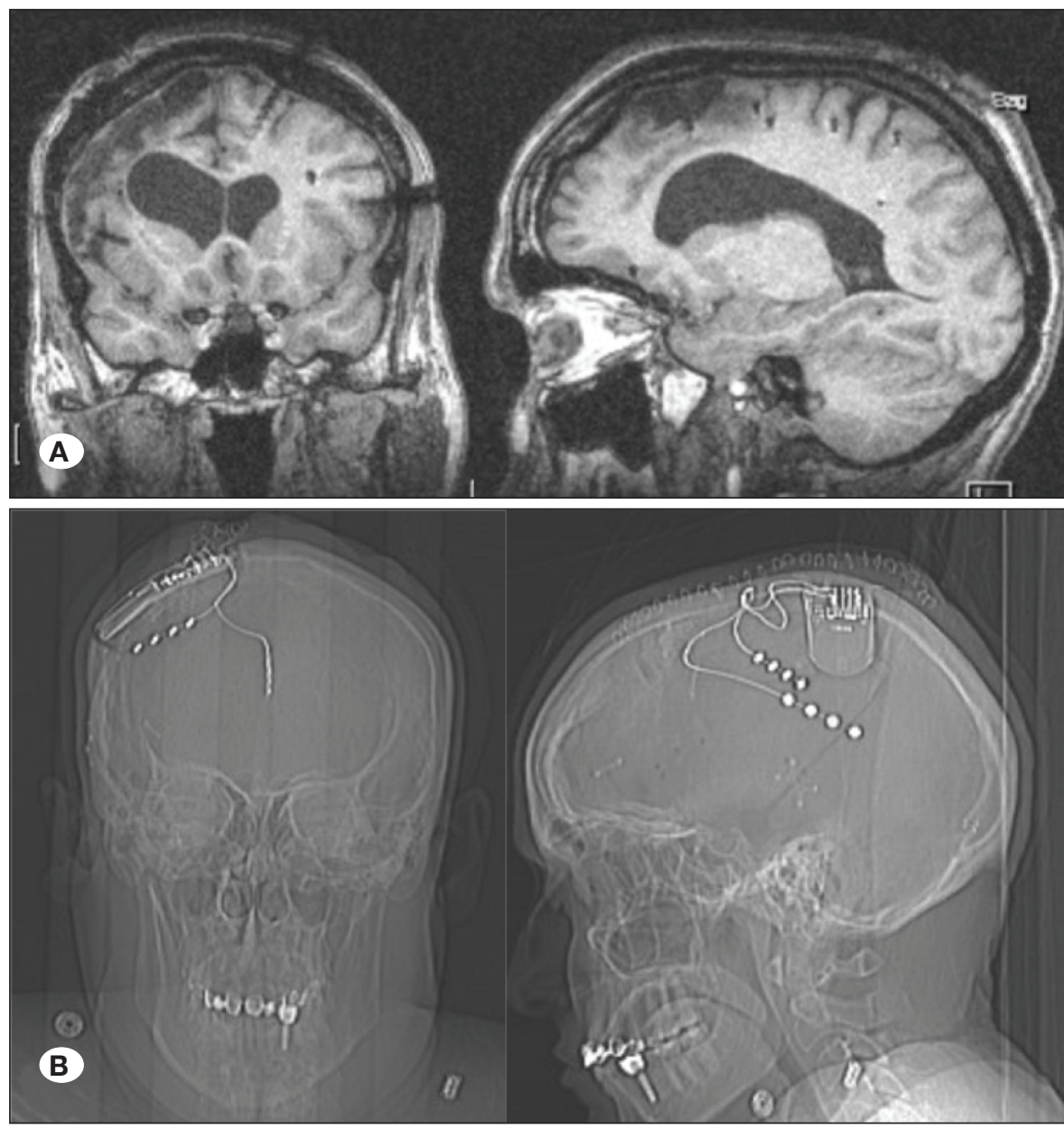

Figure 7: Cranial MRI obtained at the time of the early postoperative SEEG shows the resection cavity from the previous right lateral frontal resection and accompanying areas of encephalomalacia (A). Lateral and anteroposterior (AP) radiographs show the paddle electrodes in the interhemispheric region near the middle cingulate area and on the motor cortex after the resection of the remnant parenchyma constituting part of the $E Z(B)$.

mesial anterior cingulate areas. Her VNS system was removed along with the depth electrodes after chronic monitoring. The remnants around the previous resection cavity in the right frontal lobe were resected, and an RNS system was implanted, with two paddle electrodes in the interhemispheric region near the middle cingulate area and on the motor cortex (Figure 7B). She was seizure free at her 6-month follow-up.

\section{DISCUSSION}

We present ten patients with medically refractory epilepsy who were treated with a combination of RNS system placement and surgical resection of the EZ. In the first patient, chronic ECoG recorded by the RNS system revealed the presence of predominant epileptogenic activity in the left-sided mesial temporal region and therefore enabled a left temporal lobectomy that improved the patient's seizure outcome. This representative case suggests that patients with bilateral temporal epilepsy may benefit from the RNS system in conjunction with resection. In the remaining nine patients, EZs were either multiple in number or located in eloquent areas where surgical resection might cause irreversible neurological deficits.

RNS is a palliative seizure treatment for patients who have medically refractory epilepsy with ictal onset regions in eloquent cortical areas. RNS has been shown to be effective in patients who have bilateral disease, particularly in the mesial temporal lobe (9). A randomized controlled trial of 191 patients showed that the median reduction in seizures was $44 \%$ at 1-year follow-up and $53 \%$ at 2-year follow-up, a significant improvement over time (10). However, there are few studies demonstrating how RNS and resection can be used effectively in conjunction to maximize seizure control. To our knowledge, this is the first study that presents outcome data for patients undergoing both RNS and resection. Our results show that patients can undergo both treatment modalities safely and achieve good seizure outcomes.

Multiple studies have described the secondary benefits of the RNS system in addition to its therapeutic effect, such as providing diagnostic information regarding lateralization of seizure activity and enabling modification of subsequent resective interventions. Although RNS was designed for palliative use, there have been studies that show it can be used for chronic ambulatory ECoG. A study of 2 patients reported the successful use of RNS to localize seizure onset in patients who had nondiagnostic invasive inpatient monitoring (3). Our case series suggests that RNS can be useful for chronic ECoG and seizure localization, especially when there is a narrow range of possible locations. However, we found that RNS has limited spatial coverage, which is a limitation of 
using RNS for localization purposes. In this study, we describe a patient who underwent chronic ambulatory ECoG with RNS that also led to successful resection afterward, with excellent, albeit short-term, seizure outcomes.

There are a number of limitations to consider in this study. First, the sample size was small. Although seizure reduction was high in our patients and there were no substantial complications, the small sample size limits the power of the study and the potential inferences that can be made from it. Further studies with larger sample sizes are required to better describe outcome data and complications. Second, the follow-up period was limited and inconsistent in this study, which could influence the rate of seizure reduction observed in this patient population. However, RNS outcomes tend to improve over time; thus, our patients' seizure outcomes would likely improve with longer-term follow-up. Third, this study was performed retrospectively, and retrospective study designs carry an inherent risk of recall biases.

\section{CONCLUSION}

The RNS system has been used in conjunction with surgical resection of EZs in this small population of patients with medically intractable epilepsy when these zones were located in eloquent areas or when there was more than one zone. Here, we show that patients can tolerate undergoing both RNS and resection, achieving excellent seizure reduction and low complication rates. We also demonstrated that RNS could be used for chronic ambulatory ECoG as well as palliation. Limitations of the study include the sample size and the short duration of follow-up in some patients. Further long-term research is necessary to show the true seizure-reduction outcomes in this patient population as well as clearly define the risk and severity of potential complications.

\section{REFERENCES}

1. Ben-Menachem E, Krauss GL: Epilepsy: responsive neurostimulation-modulating the epileptic brain. Nat Rev Neurol 10:247-248, 2014

2. Bergey GK, Morrell MJ, Mizrahi EM, Goldman A, KingStephens D, Nair D, Srinivasan S, Jobst B, Gross RE, Shields DC, Barkley G, Salanova V, Olejniczak P, Cole A, Cash SS, Noe K, Wharen R, Worrell G, Murro AM, Edwards J, Duchowny M, Spencer D, Smith M, Geller E, Gwinn R, Skidmore C, Eisenschenk S, Berg M, Heck C, Van Ness P, Fountain N, Rutecki P, Massey A, O'Donovan C, Labar D, Duckrow RB, Hirsch LJ, Courtney T, Sun FT, Seale CG: Long-term treatment with responsive brain stimulation in adults with refractory partial seizures. Neurology 84:810-817, 2015

3. Chan AY, Knowlton RC, Chang EF, Rao VR: Seizure localization by chronic ambulatory electrocorticography. Clin Neurophysiol Pract 3:174-176, 2018

4. Chan AY, Mnatsakanyan L, Sazgar M, Sen-Gupta I, Lin JJ, Hsu FPK, Vadera S: Accuracy and efficacy for robotic assistance in implanting responsive neurostimulation device electrodes in bilateral mesial temporal lobe epilepsy. Oper Neurosurg (Hagerstown) 14:267-272, 2018
5. Dalkilic EB: Neurostimulation devices used in treatment of epilepsy. Curr Treat Options Neurol 19:7, 2017

6. DiLorenzo DJ, Mangubat EZ, Rossi MA, Byrne RW: Chronic unlimited recording electrocorticography-guided resective epilepsy surgery: Technology-enabled enhanced fidelity in seizure focus localization with improved surgical efficacy. J Neurosurg 120:1402-1414, 2014

7. Engel J: Surgical Treatment of Epilepsies. New York: Raven Press, 1993

8. Geller EB: Responsive neurostimulation: Review of clinical trials and insights into focal epilepsy. Epilepsy Behav 88S:1120, 2018

9. Geller EB, Skarpaas TL, Gross RE, Goodman RR, Barkley GL, Bazil CW, Berg MJ, Bergey GK, Cash SS, Cole AJ, Duckrow RB, Edwards JC, Eisenschenk S, Fessler J, Fountain NB, Goldman AM, Gwinn RP, Heck C, Herekar A, Hirsch LJ, Jobst BC, King-Stephens D, Labar DR, Leiphart JW, Marsh WR, Meador KJ, Mizrahi EM, Murro AM, Nair DR, Noe KH, Park YD, Rutecki PA, Salanova V, Sheth RD, Shields DC, Skidmore C, Smith MC, Spencer DC, Srinivasan S, Tatum W, Van Ness PC, Vossler DG, Wharen RE Jr, Worrell GA, Yoshor D, Zimmerman RS, Cicora K, Sun FT, Morrell MJ: Brain-responsive neurostimulation in patients with medically intractable mesial temporal lobe epilepsy. Epilepsia 58:9941004, 2017

10. Heck CN, King-Stephens D, Massey AD, Nair DR, Jobst BC, Barkley GL, Salanova V, Cole AJ, Smith MC, Gwinn RP, Skidmore C, Van Ness PC, Bergey GK, Park YD, Miller I, Geller E, Rutecki PA, Zimmerman R, Spencer DC, Goldman A, Edwards JC, Leiphart JW, Wharen RE, Fessler J, Fountain NB, Worrell GA, Gross RE, Eisenschenk S, Duckrow RB, Hirsch LJ, Bazil C, O'Donovan CA, Sun FT, Courtney TA, Seale CG, Morrell MJ: Two-year seizure reduction in adults with medically intractable partial onset epilepsy treated with responsive neurostimulation: Final results of the RNS System Pivotal trial. Epilepsia 55:432-441, 2014

11. King-Stephens D, Mirro E, Weber PB, Laxer KD, Van Ness PC, Salanova V, Spencer DC, Heck CN, Goldman A, Jobst B, Shields DC, Bergey GK, Eisenschenk S, Worrell GA, Rossi MA, Gross RE, Cole AJ, Sperling MR, Nair DR, Gwinn RP, Park YD, Rutecki PA, Fountain NB, Wharen RE, Hirsch LJ, Miller IO, Barkley GL, Edwards JC, Geller EB, Berg MJ, Sadler TL, Sun FT, Morrell MJ: Lateralization of mesial temporal lobe epilepsy with chronic ambulatory electrocorticography. Epilepsia 56:959-967, 2015

12. Morrell MJ, RNS System in Epilepsy Study Group: Responsive cortical stimulation for the treatment of medically intractable partial epilepsy. Neurology 77:1295-1304, 2011

13. Smart O, Rolston JD, Epstein CM, Gross RE: Hippocampal seizure-onset laterality can change over long timescales: A same-patient observation over 500 days. Epilepsy Behav Case Rep 1:56-61, 2013

14. Spencer D, Gwinn R, Salinsky M, O'Malley JP: Laterality and temporal distribution of seizures in patients with bitemporal independent seizures during a trial of responsive neurostimulation. Epilepsy Res 93:221-225, 2011 